\title{
Determinación de vida útil de harina de quinua en envase flexible
}

\author{
Determination of shelf life of quinoa flour in flexible packaging \\ ${ }^{1}$ Víctor Terry Calderón ${ }^{\mathrm{a}},{ }^{1} \mathrm{~J}$ osé Candela Díaz, ${ }^{\mathrm{a}}$ Rubén Castro Morales ${ }^{\mathrm{b}}$
}

\section{RESUMEN}

En la producción de alimentos deshidratados es necesario considerar la influencia del agua para obtener un producto con buena aceptación. El agua es un factor determinante en la inhibición o propagación de las diferentes reacciones químicas, enzimáticas o microbiológicas que pueden aumentar o reducir el valor nutritivo y la calidad de los alimentos. Una forma de correlacionar la disponibilidad de agua en los alimentos es mediante el conocimiento de su isoterma de adsorción o desorción, y el valor de capa monomolecular. Es en éste sentido que el presente estudio llegó a las siguientes conclusiones después de la evaluación del modelo matemático según GAB. La ecuación de G.A.B. presenta un mejor ajuste en la predicción de humedades de equilibrio que otras expresiones debido a que tiene en cuenta todos los puntos experimentales. Posteriormente se efectuaron experiencias para la determinación de la humedad critica $\mathrm{x}_{\mathrm{c}}(0,081 \mathrm{~g}$ de agua / $\mathrm{g}$ de sólido seco) y la humedad de equilibrio $\mathrm{x}_{\mathrm{e}}, 0,1356 \mathrm{~g}$ de agua / $\mathrm{g}$ de sólido seco) con esa base experimental se efectúa la evaluación de la harina de quinua en envase flexible, con lo cual se determino la vida útil del alimento (40 días) a una temperatura de $30{ }^{\circ} \mathrm{C}, \mathrm{HRE}: 100 \%$. Y coeficiente de permeabilidad del envase flexible.

Palabras clave: harina de quinua, determinación de vida útil, isotermas de sorción.

\begin{abstract}
In the dehydrated food production it is necessary to consider the influence of the water to obtain a product with good acceptance. The water is a determining factor in the inhibition or propagation of the different chemical reactions, enzymatic or microbiological that can increase or reduce to the nutritious value and the quality of foods. A form to correlate the water availability in foods is by means of the knowledge of its isotherm of adsorption or desorption, and the value of monomolecular layer. It is in this one sense that the present study reached the following conclusions after the evaluation of the mathematical model according to GAB. The G.A.B equation presents/displays a better adjustment in the equilibrium water prediction that other expressions because it considers all the experimental points.Later experiences for the determination of the humidity took place criticism $x_{c}(0.081$ water $g /$ dry solid $g$ ) and the equilibrium water $\mathrm{x}_{\mathrm{e}}, 0.1356$ water $\mathrm{g}$ /dry solid $\mathrm{g}$ ) with that experimental base takes place the evaluation of the flour of quinua in flexible packages, consequently I determine the life utility of the food (40 days) to a temperature of $30^{\circ} \mathrm{C}, \mathrm{HRE}: 100 \%$, value.
\end{abstract}

keyword: quinua flour, determination of life utility, isotherms of sorción.

${ }^{1}$ Universidad Nacional Federico Villareal, Lima - Perú. ${ }^{2}$ Universidad Le Cordon Bleu, Lima - Perú.

${ }^{a}$ Ingeniero Pesquero. ${ }^{b}$ Ing. Alimentario. 


\section{INTRODUCCIÓN}

Todos los alimentos incluyendo los deshidratados, contienen cierta cantidad de agua. En consecuencia, para el tecnólogo es de suma importancia conocer las propiedades físicas y químicas del agua, ya que muchas de las reacciones que suceden en los alimentos, tanto positivos como negativos, están relacionadas con la presencia de este líquido. El Tiempo de Vida Útil de un producto es el período de garantía de poder consumirlo, permaneciendo seguro y sano en las condiciones recomendadas de producción y almacenamiento. Es decir; durante el tiempo establecido como vida útil, el producto debe conservar un predeterminado nivel de calidad, bajo condiciones de almacenamiento específicas así como sus características originales de olor, sabor y textura (Casp, A, 2003).A fin de poder determinar el tiempo de vida útil, se recurre a ensayos de laboratorio, bajo condiciones controladas, monitoreando según programa diseñado, la perdida de calidad. $\mathrm{Y}$ es por ende necesaria la interpretación de los resultados, vía estadística y/o matemática, que son las herramientas vitales, que no permiten obtener modelos de simulación, con los cuales podemos pronosticar resultados a diferentes temperaturas y condiciones ambientales. (Labuza 1985). Bajo estas condiciones es necesario emplear indicadores del deterioro, es decir aquellos factores que deberán ser evaluados y controlados periódicamente, y servirán para determinar el período de vida útil, al llegar a un límite establecido, para ser considerado no apto para consumo humano, sea que el alimento haya perdido calidad por deterioro de un nutriente, o por perdida de los factores de calidad, o por desarrollo de microorganismos, generando elementos tóxicos entre otros (Casp. A, 2003).

Actualmente se encuentra en aplicación, las denominadas pruebas aceleradas también identificadas por las siglas A.S.L.T. (Acelerated Shelf Testing of Food), el cual es una de las técnicas que nos permite reducir el tiempo de determinación en los ensayos de laboratorio, para lo cual se almacenan los alimentos procesados a diferentes temperaturas, generalmente mayores a la ambiental, esto permite acelerar las reacciones químicas en los alimentos de aquellos indicadores que encuentran su desarrollo en función de la temperatura, esto genera un cierto margen de certidumbre en su determinación (Man D, 2004). En el mercado se expende mayormente harinas de cereales y de leguminosas, como son la harina de quinua, cebada, soja, de arbejas etc., las cuales se expenden a granel y sin ningún control, ni fecha de expiración, en los mercados administrados por la municipalidad. De esto se desprende que el objetivo general del presente estudio es determinar una metodología que permita realizar los ensayos respectivos para la determinación de la vida útil de este tipo de alimentos. Actualmente y gracias al avance en el conocimiento cinético de los 
diferentes procesos de deterioro, Lend (1980), así como de sus fenómenos y agentes, esta consideración en conjunto con las nuevas técnicas de análisis y la información, que permite simular el comportamiento de los sistemas altamente complejos, como es el alimento (Saguy, 1980). El objetivo del presente estudio fue la determinación las isotermas de porción del alimento a tres temperaturas, determinar la vida útil del alimento a granel y en empaque flexible determinado las ecuaciones de la cinética de deterioro.

\section{MATERIALES Y MÉTODOS}

La Investigación fue efectuada en el Laboratorio de Tecnología de los Alimentos de la Facultad de Oceanografía Pesquería y Ciencias Alimentarias, así como en las Instalaciones de la panadería de la mencionada Facultad.

Se compro la harina de quinua, en la sección cereales de la Universidad Agraria la Molina, que tiene un alto consumo popular.

\section{Materiales:}

- Equipo Kejdhal para determinar proteínas

- Crisoles y mufla, (cenizas)

- Equipo Sohxet (grasa)

- Estufa y Balanza analítica (humedad)

- Placas Conway (determinación de actividad de agua)

- Lunas de Reloj y desecadores (determinación de vida útil)

- Balanza analítica.

\section{Análisis efectuados:}

- Composición proximal de la muestra: Análisis de proteínas (método kejdhal), fibra, grasa (extracto etéreo), cenizas (método de calcinación), y humedad (método gravimétrico), empleando los materiales y equipos del laboratorio de Bioquímica de los Alimentos de la Facultad de Oceanografía, Pesquería y Ciencias alimentarias.

- Determinación de la Actividad del agua (método gravimétrico) de la harina de quinua, Labuza (1985).

- Determinación de las isotermas de porción de la harina de quinua (método gravimétrico). Materiales: Placas Conway, soluciones de sales saturadas variando de un Aw: 0.75, a un Aw: 0.75, balanza analítica, desecadores, empaque flexible y harina de quinua.

- Evaluación de la Vida útil de la harina de quinua, a granel.

- Determinación de la Vida útil de harina de quinua en empaque de polythene.

\section{Plan de trabajo:}

Análisis.

- Caracterización química de la muestra, su composición proximal.

- Determinación de las Isotermas de porción a temperatura ambiental, 30 ${ }^{\circ} \mathrm{Cy} 40^{\circ} \mathrm{C}$

- Determinar el incremento de humedad en las muestras en función del tiempo

- Determinar la humedad crítica de la harina. 
Tabla 1. Plan de trabajo

\begin{tabular}{lll}
\hline \multicolumn{1}{c}{ Etapas } & \multicolumn{1}{c}{ Tareas } \\
\hline Primera etapa & $\bullet$ & Adquisición de harina \\
& $\bullet$ & Determinación de la composición proximal (proteínas, \\
& & cenizas, humedad, grasa, y fibra) \\
& - & Determinación de la actividad de agua (aw). \\
Segunda etapa & - & Construcción de las Isotermas de sorción a tres temperaturas \\
& & $\left(20^{\circ} \mathrm{C}, 30^{\circ} \mathrm{C}\right.$ y $\left.40^{\circ} \mathrm{C}\right)$ \\
Tercera etapa & $\bullet$ & Determinar la vida útil del alimento a granel \\
Cuarta etapa & $\bullet$ & Determinar la vida útil del alimento en empaque flexible \\
Quinta etapa & $\bullet$ & Aplicación de las ecuaciones de la cinética deterioro del \\
& & alimento \\
\hline
\end{tabular}

\section{RESULTADOS}

Caracterización de la harina de quinua: Los resultados de la caracterización, mostraron que la harina con la cual se trabajo en la determinación de la vida útil tenía la siguiente composición proximal como rango de (5 determinaciones).

Tabla 2. Composición proximal de la harina de quinua con limite de confianza del $95 \%$, y actividad del agua (aw)

- $\quad$ LC $[11,49 \pm 11,68 \%]$ humedad,

- $\quad \mathrm{LC}[4,18 \pm 5,81 \%]$ grasa,

- $\mathrm{LC}[1,37 \pm 1,5 \%]$ cenizas,

- $\quad \operatorname{LC}[11,57 \pm 13,94 \%]$ proteína,

- $\quad \mathrm{LC}[2 \pm 2,9 \%]$ fibra cruda $\mathrm{y}$.

- LC $[64,16 \pm 69,26 \%]$ carbohidratos totales (determinado por diferencia),

- Con una actividad de agua (aw) de $\mathrm{LC}[0,47 \pm 0,52]$ a temperatura de $20^{\circ} \mathrm{C}$.

Las Isotermas de sorcion fueron construidas de acuerdo al método gravimétrico, considerando soluciones de sales minerales saturadas. Consignadas en la tabla 3. 
Tabla 3. Utilizadas para obtener una actividad de agua a una temperatura en grados Kelvin $(\mathrm{K})$

\begin{tabular}{|c|c|c|c|c|c|c|}
\hline Nomenclatura & Sal & & & $\begin{array}{l}\text { cuación de } \\
\text { regresión }\end{array}$ & & $\mathbf{R}^{2}$ \\
\hline LiCl & Cloruro de Litio & $\ln ($ & ) & $500,95 \frac{1}{-}$ & 3,85 & $\mathbf{0 , 9 8}$ \\
\hline $\mathrm{KC}_{2} \mathrm{H}_{3} \mathrm{O}_{2}$ & Acetato Potasio & $\ln ($ & ) & 861,39 는 & 4,33 & 0,97 \\
\hline $\mathrm{MgNO}_{3}$ & Nitrato de Magnesio & $\ln ($ & ) & 356,60 는 & 1,82 & 0,99 \\
\hline $\mathrm{NaNO}_{2}$ & Nitrato de Sodio & $\ln ($ & ) & $244,61 \frac{1}{-}$ & 0,90 & 0,90 \\
\hline
\end{tabular}

Se programo temperaturas de 30 y $40{ }^{\circ} \mathrm{C}$, lo que equivale $303{ }^{\circ} \mathrm{K}$ y $313{ }^{\circ} \mathrm{K}$, y el de medio ambiente $291^{\circ} \mathrm{K}$, obteniéndose los siguientes valores en concordancia con las ecuaciones propuestas por Labuza colocadas en la Tabla 1.

Tabla 4. Actividad de agua (aw), para soluciones de sales saturadas

\begin{tabular}{ccc}
\hline Nomenclatura & Temperaturas & (aw) \\
\hline LiCl & 291 & $\mathbf{0 , 1 2}$ \\
& 303 & 0,11 \\
$\mathrm{KC}_{2} \mathrm{H}_{3} \mathrm{O}_{2}$ & 313 & $\mathbf{0 , 1 0}$ \\
& 291 & $\mathbf{0 , 2 5}$ \\
& 303 & $\mathbf{0 , 2 2}$ \\
$\mathrm{MgNO}_{3}$ & 313 & $\mathbf{0 , 2 1}$ \\
& 291 & $\mathbf{0 , 5 5}$ \\
& 303 & $\mathbf{0 , 5 2}$ \\
$\mathrm{NaNO}_{2}$ & 313 & 0,51 \\
& 291 & 0.94 \\
& 303 & 0,91 \\
\hline
\end{tabular}

Los resultados obtenidos por vía gravimétrica, se muestran en la tabla 5. Donde se tabula temperatura y contenido de agua en función de la actividad del agua (aw).

Tabla 5. Isotermas de Sorción a $25^{\circ} \mathrm{C}, 30^{\circ}$ y $40^{\circ} \mathrm{C}$

\begin{tabular}{|c|c|c|c|c|c|}
\hline $\begin{array}{l}\text { Actividad } \\
\text { del agua } \\
\text { Aw }\end{array}$ & $\begin{array}{c}\text { Temperatura } \\
\text { (T) } 25^{\circ} \mathrm{C} \\
\text { Contenido } \\
\text { de agua (x) } \\
\text { g/g }\end{array}$ & $\begin{array}{l}\text { Actividad del } \\
\text { agua Aw }\end{array}$ & $\begin{array}{c}\text { Temperatura } \\
\text { (T) } 30^{\circ} \mathrm{C} \\
\text { Contenido } \\
\text { de agua }(\mathrm{x}) \\
\text { g/g }\end{array}$ & $\begin{array}{l}\text { Actividad } \\
\text { del agua } \\
\text { Aw }\end{array}$ & $\begin{array}{c}\text { Temperatura } \\
\text { (T) } 40^{\circ} \mathrm{C} \\
\text { Contenido } \\
\text { de agua }(\mathrm{x}) \\
\mathrm{g} / \mathrm{g}\end{array}$ \\
\hline 0.1 & 0.0373 & 0.11 & 0.0411 & 0.1200 & 0.0459 \\
\hline 0.21 & 0.0422 & 0.22 & 0.0426 & 0.2500 & 0.0490 \\
\hline 0.51 & 0.0525 & 0.52 & 0.0528 & 0.5500 & 0.0670 \\
\hline 0.89 & 0.1056 & 0.91 & 0.1065 & 0.9400 & 0.1240 \\
\hline 1 & 0.152 & 1 & 0.1680 & 1.0000 & 0.1789 \\
\hline
\end{tabular}


En las figuras 1, 3 y 5 se graficaron las isotermas de sorción. El contenido de agua (x) en función de la actividad del agua y temperatura.

En la tabla 6 y figura 2, tabla 7 y figura 4 , tabla 8 y figura 6 , se tabula de acuerdo al modelo de GAB, cuya ecuación es la siguiente. Labuza (1985):

$$
\frac{A w}{X}=\frac{1}{X m C k}+\frac{C-2}{X m C} A w+\frac{k(1-C)}{X m C} A w^{2}
$$

Y nos permite calcular el valor de la capa monomolecular de la harina de quinua.

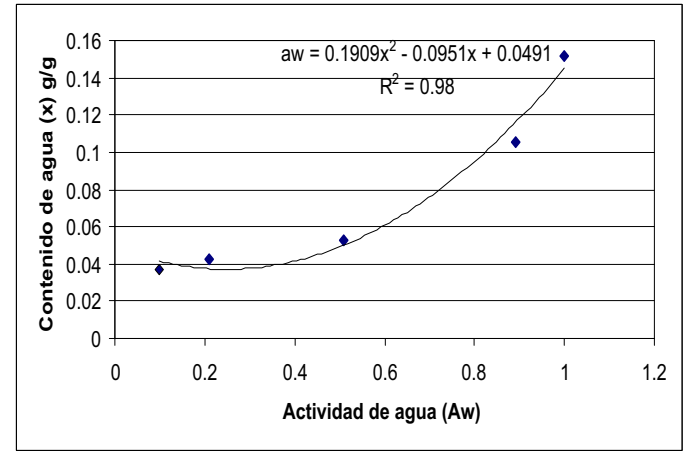

Figura 1. Isoterma de Sorción a $25^{\circ} \mathrm{C}$

Tabla 6. Aplicación de la ecuación de GAB

\begin{tabular}{rrr}
\hline \multicolumn{1}{c}{ aw } & \multicolumn{1}{c}{ x } & aw/x \\
\hline 0.1 & 0.0373 & 2.6810 \\
0.21 & 0.0422 & 4.9763 \\
0.51 & 0.0525 & 9.7143 \\
0.89 & 0.1056 & 8.4280 \\
1 & 0.152 & 6.5789 \\
\hline
\end{tabular}

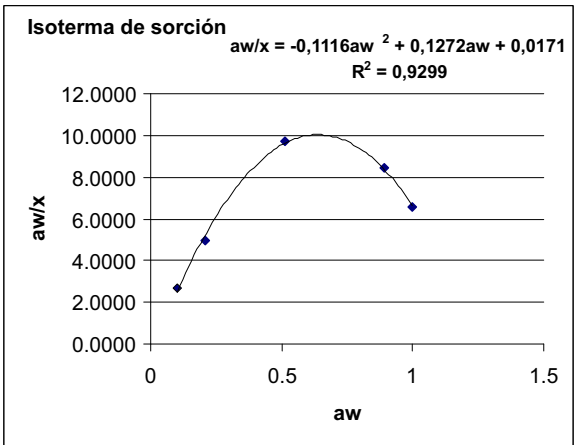

Figura 2. Isoterma de sorción según $\mathrm{GAB}$ a $25^{\circ} \mathrm{C}$

47 Rev. Tayacaja 1 (2): 42 - 53, 2018 ISSN:2617-9156

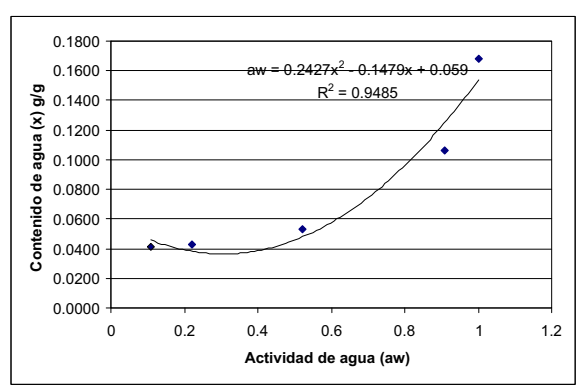

Figura 3. Isoterma de Sorcion a $30^{\circ} \mathrm{C}$

Tabla 7. Aplicación de la ecuación de GAB.

\begin{tabular}{rrr}
\hline \multicolumn{1}{c}{ Aw } & \multicolumn{1}{c}{ X } & \multicolumn{1}{c}{ aw/x } \\
\hline 0.11 & 0.0411 & 2.6764 \\
0.22 & 0.0426 & 5.1643 \\
0.52 & 0.0528 & 9.8485 \\
0.91 & 0.1065 & 8.5446 \\
1 & 0.168 & 5.9524 \\
\hline
\end{tabular}

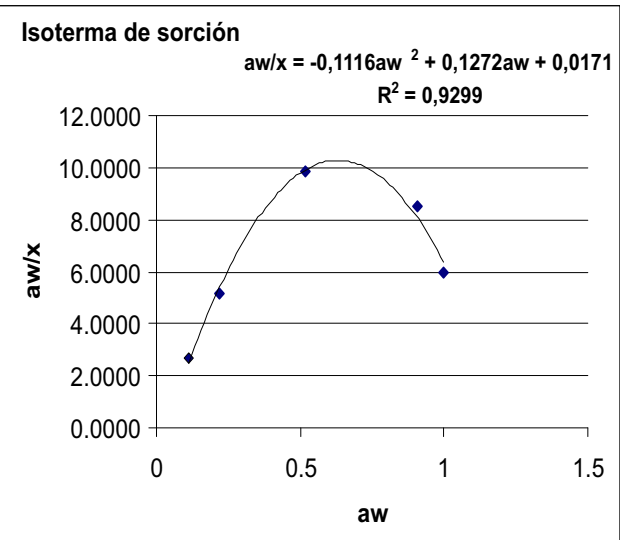

Figura 4. Isoterma de sorción según GAB a $30^{\circ} \mathrm{C}$

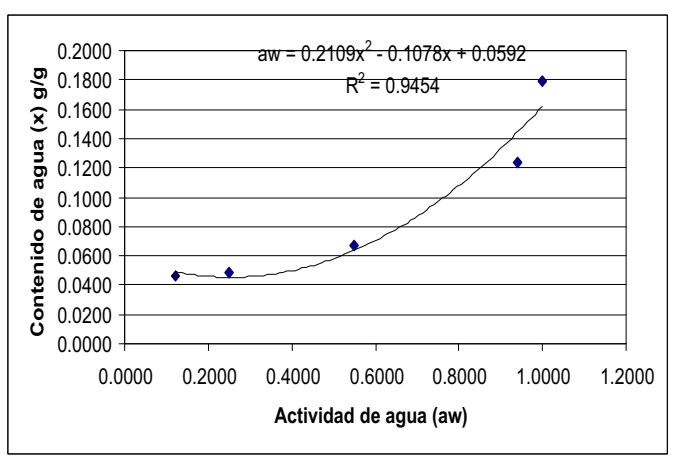

Figura 5. Isoterma de Sorción a $40^{\circ} \mathrm{C}$ 
Tabla 8. Aplicación de la Ecuación de GAB

\begin{tabular}{rrr}
\hline \multicolumn{1}{c}{ Aw } & \multicolumn{1}{c}{$\mathrm{x}$} & \multicolumn{1}{c}{$\mathrm{aw} / \mathrm{x}$} \\
\hline 0.12 & 0.0459 & 2.6144 \\
0.25 & 0.049 & 5.1020 \\
0.55 & 0.067 & 8.2090 \\
0.94 & 0.124 & 7.5806 \\
1 & 0.168 & 0.1789 \\
\hline
\end{tabular}

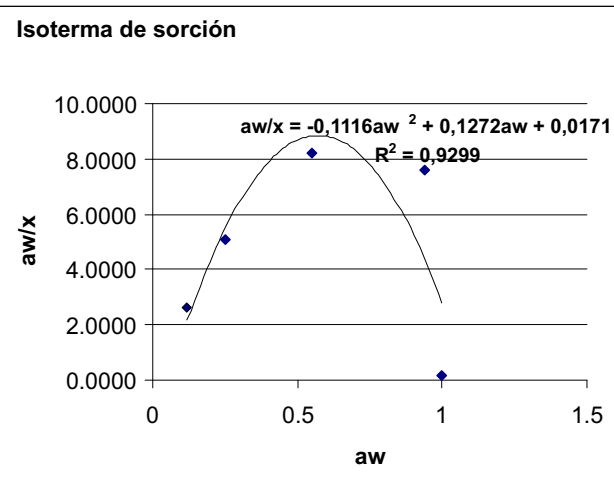

Figura 6. Isoterma de sorción según GAB a $40{ }^{\circ} \mathrm{C}$.

Deterioro de la harina de quinua a granel

Se colocaron en 02 desecadores muestra de harina de quinua deshidratada, 25 gramos, (en estufa hasta peso constante), en lunas de reloj, colocando en la base del desecador agua destilada lo cual representa un HRE: 100\%. Los resultados se tabulan en la tabla N 9, y la representación gráfica en las figuras 7, 8 y 9.

Tabla 9. Ganancia de agua $\left(\mathrm{W}_{\mathrm{i}}\right)$

\begin{tabular}{cccc}
\hline $\begin{array}{c}\text { Tiempo } \\
\text { (t) día }\end{array}$ & $\begin{array}{c}\text { Ganancia de } \\
\text { peso w (g) }\end{array}$ & $\begin{array}{c}\text { Ganancia de } \\
\text { peso w1(g) }\end{array}$ & $\begin{array}{c}\text { Ganancia de } \\
\text { peso w2(g) }\end{array}$ \\
\hline 0 & 0 & 0 & 0 \\
2 & 1.11 & 1.12 & 1.15 \\
3 & 1.42 & 1.43 & 1.42 \\
4 & 1.48 & 1.64 & 1.61 \\
7 & 1.82 & 1.91 & 1.92 \\
10 & 1.82 & 1.91 & 1.92 \\
\hline
\end{tabular}

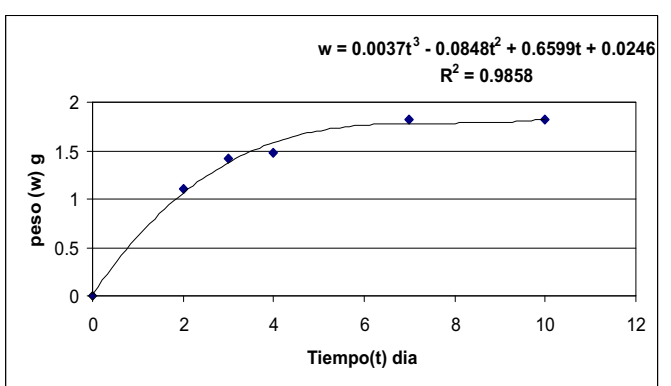

Figura 7. Ganancia de agua (w) en función del tiempo (t)

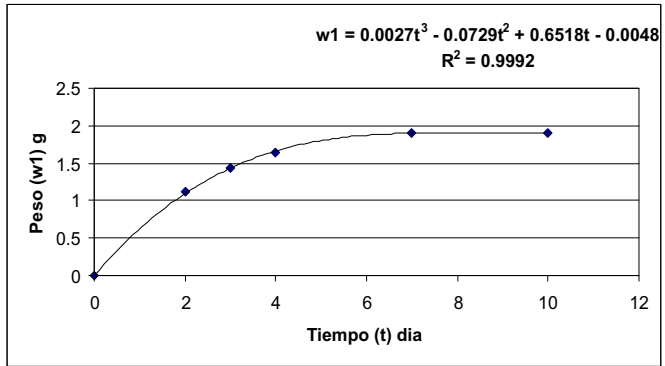

Figura 8. Ganancia de agua (w1) en función del tiempo (t)

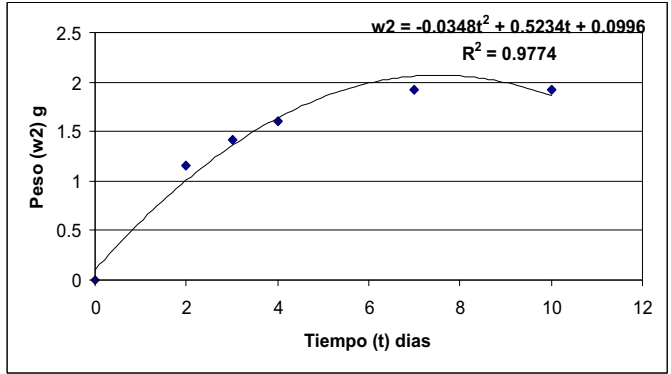

Figura 9. Ganancia de agua (w2) en función del tiempo (t)

Cuando la humedad alcanza entre el 10 al $12 \%$, harina empieza a formar grumos posteriormente comienza la proliferación de microorganismos. Como se puede apreciar en las fotos.

Determinación del tiempo de vida útil en envase flexible (Lend 1980)

Basada en la información de las experiencias realizadas anteriormente se obtuvieron los siguientes valores, que se aplicaron a la experiencia. 
Tabla 10. Condiciones iniciales para la determinación de vida útil en envase flexible

\begin{tabular}{|ll|}
\hline Humedad inicial & $\mathrm{x}_{\mathrm{o}}=0,045 \mathrm{~g}$ de agua $/ \mathrm{g}$ de sólido seco \\
Humedad critica & $\mathrm{x}_{\mathrm{c}}=0,11 \mathrm{~g}$ de agua $/ \mathrm{g}$ de sólido seco \\
Coeficiente de sorción & $\mathrm{x}_{\mathrm{e}}=0,1356 \mathrm{~g}$ de agua $/ \mathrm{g}$ de sólido seco \\
Area de empaque & $\alpha=0,182$ \\
Masa de sólidos seco & $\mathrm{A}=28 \mathrm{~g}$ \\
Presión de vapor & $\mathrm{Po}=23,75 \mathrm{~mm}$ de mercurio \\
\hline
\end{tabular}

Coeficiente de permeabilidad del envase:

$$
\frac{K m}{L} \quad \frac{g m}{\operatorname{diam} \frac{m m H g}{m}}
$$

El modelo matemático que interpreta el fenómeno es:

$$
\operatorname{Ln} \frac{x \quad x}{x \quad x} \quad \frac{K m}{L} \quad \frac{A P o}{M} t
$$

Extrayendo el antilogaritmo:

$$
\left(\frac{x_{e}-x_{0}}{x_{e}-x_{c}}\right)=e^{\left(\frac{K m}{L}\right) \cdot\left[\frac{A \cdot P o}{M \cdot \alpha}\right] \cdot t}
$$

El puede reemplazase el valor $\mathrm{x} \rightarrow \mathrm{x}_{\mathrm{c}}$ Y el coeficiente:

$$
\left(\frac{K m}{L}\right) \cdot\left[\frac{A \cdot P o}{M \cdot \alpha}\right]=\beta
$$

Reemplazando se obtiene:

$$
\left(\frac{x_{e}-x_{0}}{x_{e}-x}\right)=e^{\beta . t}
$$

Invirtiendo la expresión se obtiene:

$$
\left(\frac{x_{e}-x}{x_{e}-x_{o}}\right)=e^{-\beta . t}
$$

Para determinar la vida útil de la harina de quinua en un envase permeable se efectúo la siguiente programación de las experiencias (03). Se programaron 3 desecadores conteniendo agua destilada $(\mathrm{Aw}=1$, equivalente a $\mathrm{HRE}=1 \%)$, donde se colocaron (06) empaques conteniendo harina de quinua, estos desecadores fueron posteriormente colocados en una estufa, a una temperatura programada de $30{ }^{\circ} \mathrm{C}$, y cada periodo de tiempo se fueron

\begin{tabular}{|c|c|c|c|c|c|}
\hline $\begin{array}{l}\text { Tiempo(t) } \\
\text { días }\end{array}$ & $\begin{array}{c}\text { Ganancia } \\
\text { de peso } \\
\mathrm{X}_{1}, \\
(\mathrm{~g} / \mathrm{g})\end{array}$ & $\begin{array}{l}\text { Ganancia } \\
\text { de peso } X_{2} \text {, } \\
(\mathrm{g} / \mathrm{g})\end{array}$ & $\begin{array}{c}\text { Ganancia } \\
\text { de peso } \\
X_{3},(g / g)\end{array}$ & $\begin{array}{c}\text { Ganancia } \\
\text { de peso } \\
\text { promedio } \\
(\mathrm{g} / \mathrm{g})\end{array}$ & $\begin{array}{c}\text { Coeficiente } \\
\text { de } \\
\text { humedad } \\
\text { (u) }\end{array}$ \\
\hline 0 & 0.045 & 0.045 & 0.045 & 0.045 & 1 \\
\hline 20 & 0.068 & 0.066 & 0.067 & 0.067 & 0.751 \\
\hline 40 & 0.086 & 0.084 & 0.084 & 0.085 & 0.552 \\
\hline 60 & 0.100 & 0.098 & 0.098 & 0.099 & 0.396 \\
\hline 80 & 0.108 & 0.105 & 0.106 & 0.106 & 0.309 \\
\hline 100 & 0.116 & 0.114 & 0.114 & 0.115 & 0.215 \\
\hline 120 & 0.120 & 0.118 & 0.118 & 0.119 & 0.171 \\
\hline 140 & 0.126 & 0.124 & 0.124 & 0.125 & 0.102 \\
\hline 160 & 0.127 & 0.124 & 0.125 & 0.125 & 0.095 \\
\hline 180 & 0.130 & 0.127 & 0.128 & 0.128 & 0.064 \\
\hline
\end{tabular}
pesando para observar el incremento de peso, el tiempo programado fue de 120 días. Los valores obtenidos están tabulados en la tabla N 11.

Tabla 11. Ganancia de peso $\left(x_{i}\right)$ g/g de sólido seco en envase flexible a $30{ }^{\circ} \mathrm{C}$ y un

HRE : $100 \%$ 
De acuerdo a los estudios efectuados la humedad critica se alcanza en 80 días, es decir $\mathrm{x}=0,11 \mathrm{~g}$ de agua $/ \mathrm{g}$ de sólidos seco, lo cual representa la vida útil del alimento en envase flexible del tipo polythene, con un espesor de 1 mil.

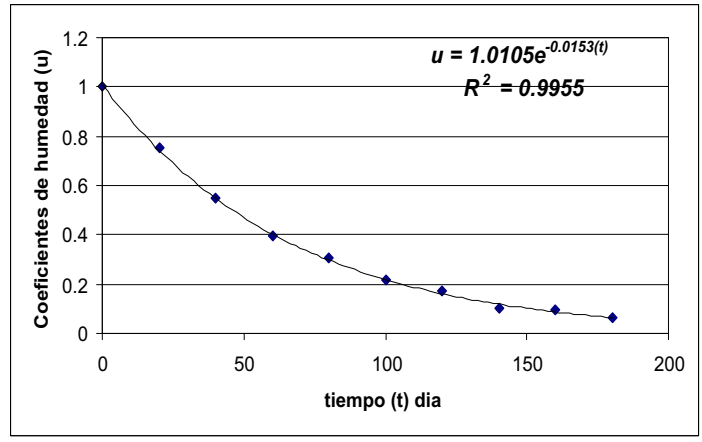

Figura 11. Coeficientes de humedad (u) en función del tiempo.

La ecuación que permite predecir el tiempo de vida útil para las condiciones de la experiencia programada es:

$$
\begin{gathered}
x=x_{e}-1,010 \cdot\left(x_{e}-x_{o}\right) e^{-0,0153(t)} \\
\left(R^{2}=0,9955\right)
\end{gathered}
$$

Donde:

$\mathrm{x}$ : el contenido de humedad ( $\mathrm{g}$ de agua / $\mathrm{g}$ de sólido seco) en un tiempo ( $\mathrm{t}$ ) días $\mathrm{x}_{\mathrm{e}}$ : Contenido de humedad en el equilibrio ( $\mathrm{g}$ de agua/ $\mathrm{g}$ de sólido seco) $\mathrm{x}_{\mathrm{o}}$ : contenido de humedad inicial ( $\mathrm{g}$ de agua / g de sólido seco)

$t$ : tiempo en días

\section{DISCUSION}

Los resultados obtenidos se basan fundamentalmente en las consideraciones cinéticas propuestas por Labuza (1985), determinadas por las denominadas pruebas aceleradas, En donde el deterioro se ve influenciada por la temperatura. $Y$ es en este caso como la temperatura favorece la absorción del agua por parte de la harina de quinua motivando su posterior deterioro.

El incremento de agua en las pruebas relacionadas con la determinación de la vida útil de los alimentos (Saguy 1980), se ven sustentadas por la ecuación que sirve de base para determinar la ecuación que predice el tiempo de vida útil de la harina de quinua en envase de polythene.

\section{CONCLUSIONES}

De acuerdo a los estudios efectuados se tiene la siguiente secuencia del trabajo que permite llegar a los valores planteados en los objetivos:

- Composición proximal de la harina de quinua, determinando contenido de humedad, proteínas, cenizas, fibra cruda, grasa y por diferencia hidratos de carbono.

- Grafica de las isotermas de sorción, mostrando el contenido de humedad con su respectiva actividad de agua a tres temperaturas diferentes, cuando se emplea la ecuación de GAB, permite determinar el contenido de la capa monomolecular cuyo valor se obtiene a partir de la ecuación de GAB obtenida por análisis de regresión (4.17 $\mathrm{g} \mathrm{H}_{2} \mathrm{O} / 100 \mathrm{~g}$ de sólido seco).

- Estudio del deterioro de la harina de quinua, colocadas en desecadores, conteniendo agua destilada que simula condiciones desfavorables de almacenamiento (HRE:100\%).

- El contenido de humedad critico, obtenido por vía organoléptica, esta 
dentro de los contenidos de humedad del 10 al $11 \%$, donde se muestra un aglomeramiento de la harina de quinua, sometida a un HRE :100\%.

- Se determino la vida útil del alimento (harina de quinua) envasada en empaque flexible de polythene, con un espesor de 1 mil, obteniéndose la ecuación pertinente que permite calcular el contenido de humedad de la muestra en función del tiempo. El tiempo de vida útil del alimento en el empaque flexible fue de 80 días.

\section{REFERENCIASBIBLIOGRÁFICAS}

Casp, a, (2003) PROCESOS DE CONSERVACION DE ALIMENTOS, ed. acribia, segunda edición, España.

Man D, (2004), CADUCIDAD DE LOS ALIMENTOS, primera edición, Science, South Bank University, London.

Potter, N (1999), CIENCIA DE LOS ALIMENTOS Editorial Acribia, España.

$\mathrm{G}$ o $1 \mathrm{~d}$ m a n, $\mathrm{K} \quad\left(\begin{array}{llll}1 & 9 & 8 & 3\end{array}\right)$. DECOLORIZATION OF (BCAROTENE IN MODEL SYSTEM SIMULATING DEHYDRATED FOODS. MECHANISMS AND KINETIC PRINCIPLES. J. Food Sci., v. 48, n.3, p.751-754.

Labuza (1985) Accelerated Shelf life testing of foods. Food Technol., v.39,n 9, pag $57-64$.
Saguy (1980) Modeling of quality deterioration during food processing and storage. Food Technol., vol 34,n2, p.51 -54.

Lend (1980) Experimental procedures for determining destruction kinetics of food components. Food Technol.,vol34,n 3, pag 35-40.

\section{CORRESPONDENCIA}

Dr. Victor Terry Calderón victor.terry@ulcb.edu.pe

\section{ANEXOS}

Fotografía 1. Muestra de Harina de quinua deshidratada hasta peso constante y colocado en placas petri.

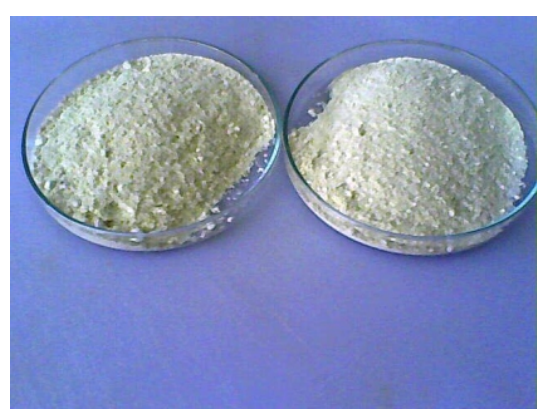

Fotografía 2. Muestras Harina de quinua colocada en lunas de reloj y puesta en un desecador.

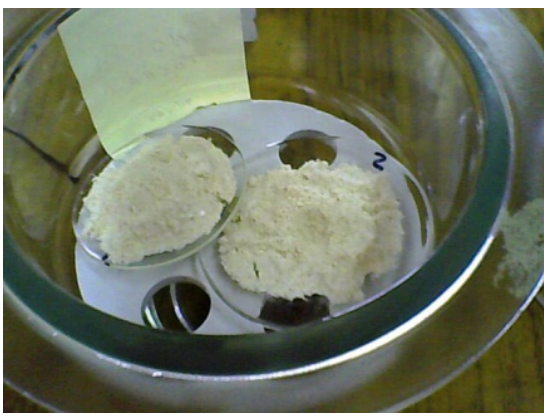


Fotografía 3. Muestras Harina de quinua colocada en lunas de reloj y puesta en un desecador.

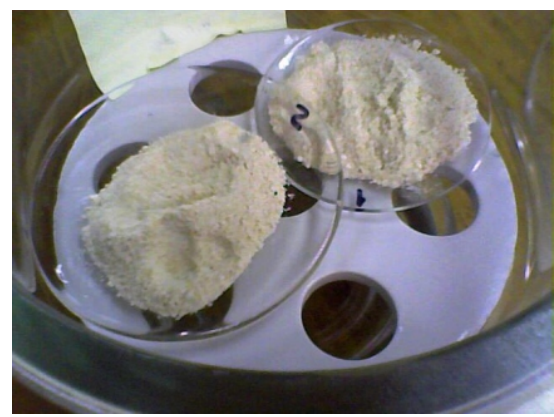

Fotografía 4. Muestras de Harina Colocada en Desecador, en cuya cámara se colocó agua destilada.

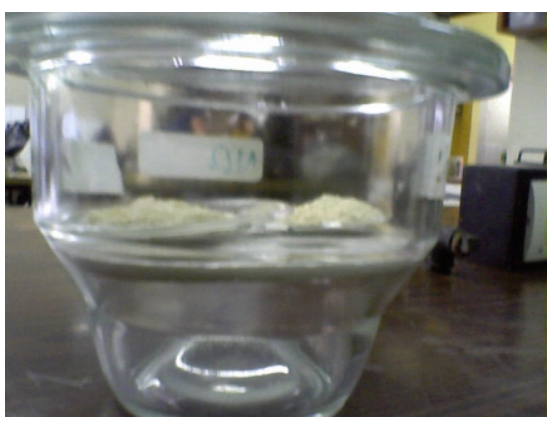

Figura 5. Muestras de Harina de qu in u a, mos t r a n d o aglomeraciones y comienzo de deterioro.

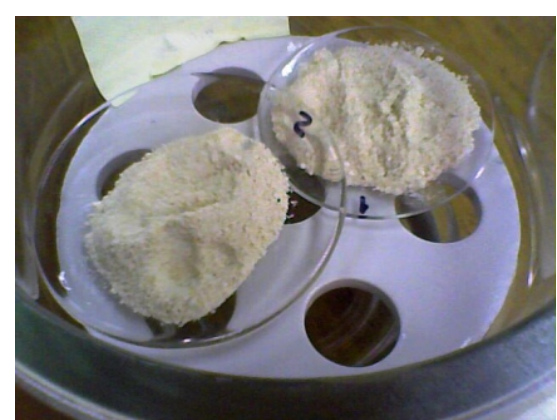

Figura 6. Muestras de Harina Colocada en Desecador, en proceso de deterioro.

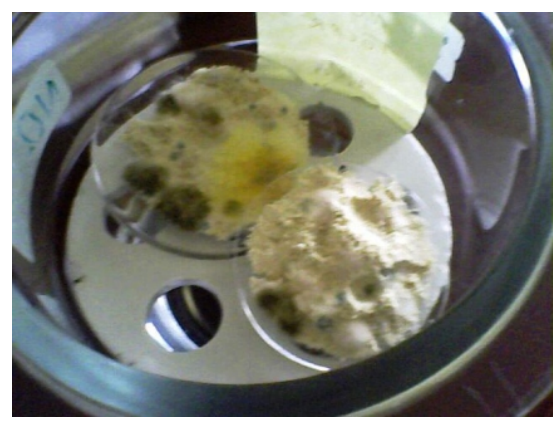

Figura 7. Muestras de Harina Colocada en Desecador, mostrado deterioro por efecto de a humedad.
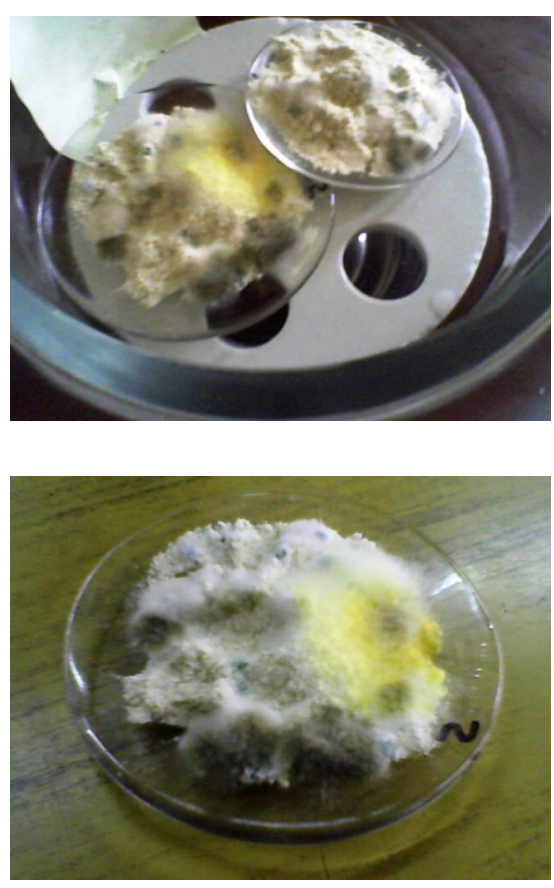
Figura 9. Muestras de Harina Colocada en Desecador, mostrando deterioro.

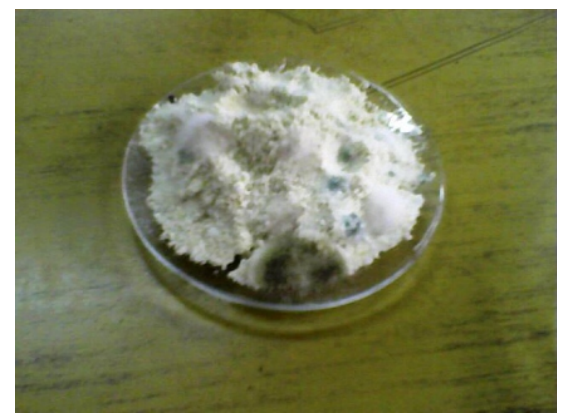

Fotografía 10. Muestras de Harina en envases y colocada en Desecador para determinar la vida útil del alimento en empaque flexible.

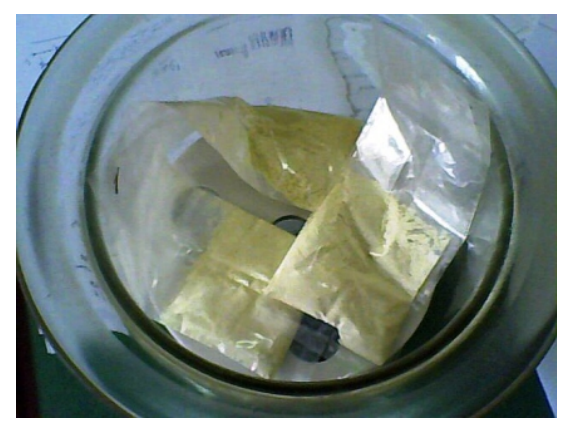

Figura 11. Muestras de Harina en envases y colocada en Desecador, para determinar la vida útil del alimento en empaque flexible, la cámara inferior esta con agua destilada.

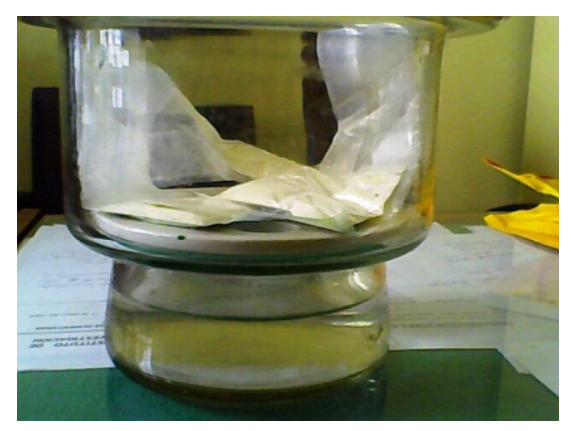

